\title{
ON THE KERNEL OF HOLONOMY
}

\author{
A. P. Caetano
}

\begin{abstract}
A connection on a principal $G$-bundle may be identified with a smooth group morphism $\mathcal{H}: \mathcal{G L}^{\infty}(M) \rightarrow G$, called a holonomy, where $\mathcal{G} \mathcal{L}^{\infty}(M)$ is a group of equivalence classes of loops on the base $M$. The present article focuses on the kernel of this morphism, which consists of the classes of loops along which parallel transport is trivial. Use is made of a formula expressing the gauge potential as a suitable derivative of the holonomy, allowing a different proof of a theorem of Lewandowski's, which states that the kernel of the holonomy contains all the information about the corresponding connection. Some remarks are made about nonsmooth holonomies in the context of quantum Yang-Mills theories.
\end{abstract}

\section{Introduction}

In differential geometry one associates to every connection defined on a principal $G$-bundle, together with a choice of base point in the bundle, a holonomy, i.e. a map from a space of piecewise smooth loops to the group $G$. Holonomy describes parallel transport along these loops. In what follows we first present a summary of our approach to exhibiting holonomy as a morphism from a group of loops to $G$ (Section 2). In Section 3 we derive a formula which expresses the gauge potential as a suitable derivative of the holonomy. Along with the Ambrose-Singer theorem, this formula plays a central role in our main result: a proof within our approach to a theorem due to Lewandowski $[\mathbf{L}]$, which states that the kernel of the holonomy contains all the information about the corresponding connection (Section 4). In Section 5 we add some remarks on the regularity of the images of generalized holonomies. 


\section{Holonomy and the group of loops}

Let $M$ be a smooth, connected and paracompact manifold and $* \in M$, a fixed point. Following $[\mathbf{C P}]$ we denote by $P^{\infty}(M)$ the space of all smooth paths in $M$ having 0 and 1 as sitting instants, that is, paths which stop for a while at their extremities. Every piecewise smooth path may be reparametrized to become a member of this space. $P^{\infty}(M, *)$ shall be the subspace of $P^{\infty}(M)$ consisting of paths which start at $* \in M$ and $\Omega^{\infty}(M)$ shall be the subspace of $P^{\infty}(M, *)$ consisting of loops.

Given two paths $l, k \in P^{\infty}(M)$ we say that they are intimate, and write $l \stackrel{\rho}{\sim} k$, if there exists a smooth homotopy $H:[0,1] \times[0,1] \rightarrow M$ with $\operatorname{rank} \operatorname{rk}\left(D H_{(s, t)}\right) \leq 1, \forall(s, t) \in[0,1] \times[0,1]$ and for which there is $0<\epsilon<$ $1 / 2$ such that $0 \leq s \leq \epsilon \Rightarrow H(s, t)=l(t), 1-\epsilon \leq s \leq 1 \Rightarrow H(s, t)=k(t)$, $0 \leq t \leq \epsilon \Rightarrow H(s, t)=l(0)$ and $1-\epsilon \leq t \leq 1 \Rightarrow H(s, t)=l(1)$. Such a map $H$ will be called a rank-one-homotopy. The usual operations of composition, inversion and reparametrization of paths go over to the quotient by intimacy to give rise to an "algebra" of classes of paths where associativity holds, trivial paths act as identities and "tails" of the form $l l^{-1}$ may be included or thrown away. Restricting the operations with paths to $\Omega^{\infty}(M)$ these results show that the quotient $\Omega^{\infty}(M) / \stackrel{\rho}{\sim}$ becomes a group. This "group of loops", denoted by $\mathcal{G} \mathcal{L}^{\infty}(M)$, is a topological invariant for smooth manifolds.

Given a Lie group $G$ and a principal $G$-bundle $\pi: P \rightarrow M$ equipped with a connection $\nabla$ one can perform parallel transport along each loop $l \in \Omega^{\infty}(M)$. Together with the right $G$-action on $P$ this operation defines the holonomy $\mathcal{H}: \Omega^{\infty}(M) \longrightarrow G$ in the following way: let $l^{\uparrow}:[0,1] \rightarrow P$ be the unique horizontal lift of $l$ such that $l^{\uparrow}(0)$ is a fixed point; then $\mathcal{H}(l)$ is the unique element of $G$ satisfying the equation $l^{\uparrow}(0)=l^{\uparrow}(1) \mathcal{H}(l)$. The holonomy goes over to the quotient by intimacy to define a group morphism $\mathcal{H}_{\nabla}: \mathcal{G} \mathcal{L}^{\infty}(M) \rightarrow G$. This result is the initial motivation for "redefining" holonomy in $[\mathbf{C P}]$ :

Definition 1. A holonomy is a group morphism $\mathcal{H}: \mathcal{G} \mathcal{L}^{\infty}(M) \rightarrow G$ such that for every smooth family of loops $\psi: U \subseteq \mathbf{R}^{n} \rightarrow \Omega^{\infty}(M)$ the composition

$$
\mathbf{R}^{n} \supset U \stackrel{\psi}{\longrightarrow} \Omega^{\infty}(M) \stackrel{\text { proj }}{\longrightarrow} \mathcal{G} \mathcal{L}^{\infty}(M) \stackrel{\mathcal{H}}{\longrightarrow} G
$$

where proj is the natural projection, is smooth throughout the open subset $U \subseteq R^{n}$.

Theorem 1. There is a one-to-one correspondence between holonomies and triples consisting of a principal G-bundle, a connection on this bundle and a point in the fiber over *, up to isomorphism. 
This result is obtained through the reconstruction of a bundle and a connection from a holonomy $\mathcal{H}: \mathcal{G} \mathcal{L}^{\infty}(M) \rightarrow G$. The set which becomes the reconstructed bundle is the quotient of $P^{\infty}(M, *) \times G$ by the equivalence relation

$$
(p, g) \sim(q, h) \text { iff } p(1)=q(1) \wedge h=\mathcal{H}\left(q p^{-1}\right) g
$$

The equivalence class of $(p, g) \in P^{\infty}(M, *) \times G$ is denoted by $[(p, g)]$. $B$ is the set of equivalence classes. There is a natural projection $\pi$ : $B \rightarrow M$ given by $\pi([(p, g)])=p(1)$ and a natural free right action of $G$, given by $[(p, g)] h=[(p, g h)]$. The reconstruction of a connection follows naturally from the possibility of lifting a path: given $q \in P^{\infty}(M, *)$ let $q_{k} \in P^{\infty}(M, *)$ for each $k \in[0,1]$ be the restriction of $q$ to $[0, k]$, suitably reparametrized. Then for each $g \in G$ a lift of $q$ is defined by $[0,1] \ni k \mapsto\left[\left(q_{k}, g\right)\right] \in B$ and this is a horizontal lift of the reconstructed connection.

\section{Holonomy and gauge potentials}

We now briefly describe the local trivializations for the reconstructed bundle. After choosing an atlas for $M$ consisting of charts $\phi_{\alpha}: U_{\alpha} \subseteq$ $M \rightarrow \mathbf{R}^{n}$ whose images are the unit open ball $B(\overrightarrow{0}, 1)$, the inverse retraction of this ball may be transported to each $U_{\alpha}$ by means of the corresponding $\phi_{\alpha}$. This permits us to associate to each $m \in U_{\alpha}$ a path $\gamma_{\alpha}^{m} \in P^{\infty}(M)$ which starts at $\phi_{\alpha}^{-1}(\overrightarrow{0})$ and ends at $m \in U_{\alpha}$. Fixing a path $p \in P^{\infty}(M, *)$ such that $p(1)=\phi_{\alpha}^{-1}(\overrightarrow{0})$, the elements of $\pi^{-1}\left(U_{\alpha}\right)$ may be written down in the form $\left[\left(p \gamma_{\alpha}^{m}, g\right)\right]$ so that the equality $T_{\alpha}\left(\left[\left(p \gamma_{\alpha}^{m}, g\right)\right]\right)=(m, g)$ establishes a bijection $T_{\alpha}: \pi^{-1}\left(U_{\alpha}\right) \rightarrow U_{\alpha} \times G$ that is compatible with the right- $G$ action on $B$. $T_{\alpha}$ acts as a local G-bundle isomorphism.

The reconstructed connection $\nabla$ is carried by $T_{\alpha}$ to become $\hat{\nabla}=$ $\left(T_{\alpha}^{-1}\right)^{*} \nabla$. For this consider the equivariant Lie algebra valued 1-form $\omega \in \bigwedge^{1}\left(\pi^{-1}\left(U_{\alpha}\right)\right) \otimes \mathcal{G}$ defined by $\nabla$ and take $\hat{\omega}=\left(T_{\alpha}^{-1}\right)^{*} \omega \in \bigwedge^{1}\left(U_{\alpha} \times\right.$ $G) \otimes \mathcal{G}$. The canonical section of $U_{\alpha} \times G$ defined by $r_{\alpha}(x)=(x, \mathbf{e})$ where $\mathbf{e}$ is the identity of the Lie group, corresponds to a section of $\pi^{-1}\left(U_{\alpha}\right)$ given by $s_{\alpha}(x)=T_{\alpha}^{-1}\left(r_{\alpha}(x)\right)$. The 1 -form $\Lambda_{\alpha} \in \bigwedge^{1}\left(U_{\alpha}\right) \otimes \mathcal{G}$ given by $\Lambda_{\alpha}=s_{\alpha}^{*} \omega=r_{\alpha}^{*} \hat{\omega}$ is a local gauge potential for $\nabla$ and $\hat{\nabla}$.

Now we shall look for a formula expressing the gauge potential $\Lambda_{\alpha}$ directly in terms of the holonomy $\mathcal{H}: \mathcal{G L}^{\infty}(M) \rightarrow G$. Returning to the framework of the local trivialization $T_{\alpha}$ we consider an arbitrary point $m \in U_{\alpha}$ and a path $q \in P^{\infty}(M, *)$ such that $q(a)=m$ for some $a \in] 0,1\left[\right.$. For each $k \in[0,1], q_{k} \in P^{\infty}(M, *)$ is the restriction of $q$ 
to $[0, k]$, suitably reparametrized. Taking into account the equivalence relation on $P^{\infty}(M, *) \times G$ it's easy to check that the following curves

$$
\begin{aligned}
& {[0,1] \ni k \longmapsto T_{\alpha}\left[\left(q_{k}, \mathcal{H}\left(q_{a} \gamma_{\alpha}^{m^{-1}} p^{-1}\right)\right)\right] \in U_{\alpha} \times G} \\
& {[0,1] \ni k \longmapsto T_{\alpha}\left[\left(p \gamma_{\alpha}^{q(k)}, \mathcal{H}\left(p \gamma_{\alpha}^{q(k)} q_{k}^{-1} q_{a} \gamma_{\alpha}^{m^{-1}} p^{-1}\right)\right)\right] \in U_{\alpha} \times G}
\end{aligned}
$$

are actually the same curve and, since the intimacy relation "disregards tails", it may be further expressed, for $k$ close to $a$, as

$$
[0,1] \ni k \longmapsto T_{\alpha}\left[\left(p \gamma_{\alpha}^{q(k)}, \mathcal{H}\left(p \gamma_{\alpha}^{q(k)} q_{\mid[k, a]} \gamma_{\alpha}^{m^{-1}} p^{-1}\right)\right)\right] \in U_{\alpha} \times G
$$

where $q_{\mid[k, a]}$ is to be read as $q_{\mid[a, k]}{ }^{-1}$ whenever $a \leq k$. This curve goes through the point $r_{\alpha}(m)=(m, \mathbf{e})$ when $k=a$, and is by construction a horizontal lift of $q$ with respect to $\hat{\nabla}$, so that

$$
\hat{\omega}_{(m, \mathbf{e})}\left(\frac{d}{d k}{ }_{\mid k=a} T_{\alpha}\left[\left(p \gamma_{\alpha}^{q(k)}, \mathcal{H}\left(p \gamma_{\alpha}^{q(k)} q_{\mid[k, a]} \gamma_{\alpha}^{m^{-1}} p^{-1}\right)\right)\right]\right)=0 .
$$

These remarks justify the following calculations:

$$
\begin{aligned}
\Lambda_{\alpha}(m)\left(\frac{d q}{d k}(a)\right)= & r_{\alpha}^{*} \hat{\omega}\left(\frac{d q}{d k}(a)\right) \\
= & \hat{\omega}_{r_{\alpha}(m)}\left(D r_{\alpha}(m)\left(\frac{d q}{d k}(a)\right)\right) \\
= & \hat{\omega}_{(m, \mathbf{e})}\left(\frac{d q}{d k}(a), 0\right) \\
= & \hat{\omega}_{(m, \mathbf{e})}\left(\left(\frac{d q}{d k}(a), \frac{d}{d k}_{\mid k=a} \mathcal{H}\left(p \gamma_{\alpha}^{q(k)} q_{\mid[k, a]} \gamma_{\alpha}^{m^{-1}} p^{-1}\right)\right)\right. \\
& \left.+\left(0,-\frac{d}{d k}{ }_{\mid k=a} \mathcal{H}\left(p \gamma_{\alpha}^{q(k)} q_{\mid[k, a]} \gamma_{\alpha}^{m^{-1}} p^{-1}\right)\right)\right) \\
= & \hat{\omega}_{(m, \mathbf{e})}\left(\frac{d}{d k}{ }_{\mid k=a} T_{\alpha}\left[\left(p \gamma_{\alpha}^{q(k)}, \mathcal{H}\left(p \gamma_{\alpha}^{q(k)} q_{\mid[k, a]} \gamma_{\alpha}^{m^{-1}} p^{-1}\right)\right)\right]\right) \\
& +\hat{\omega}_{(m, \mathbf{e})}\left(0,-\frac{d}{d k}{ }_{\mid k=a} \mathcal{H}\left(p \gamma_{\alpha}^{q(k)} q_{\mid[k, a]} \gamma_{\alpha}^{m^{-1}} p^{-1}\right)\right) \\
= & \hat{\omega}_{(m, \mathbf{e})}\left(0,-\frac{d}{d k}{ }_{\mid k=a} \mathcal{H}\left(p \gamma_{\alpha}^{q(k)} q_{\mid[k, a]} \gamma_{\alpha}^{m^{-1}} p^{-1}\right)\right) \\
= & -\frac{d}{d k}{ }_{\mid k=a} \mathcal{H}\left(p \gamma_{\alpha}^{q(k)} q_{\mid[k, a]} \gamma_{\alpha}^{m^{-1}} p^{-1}\right)
\end{aligned}
$$

where the last equality comes from the compatibility between the connection 1-form $\hat{\omega}$ and the right $G$-action of $G$ on $U_{\alpha} \times G$. The differential 
of $G \ni x \longmapsto x^{-1} \in G$ at $\mathbf{e} \in G$ is $\mathcal{G} \ni u \longmapsto-u \in \mathcal{G}$ so that the final formula is:

$$
\Lambda_{\alpha}(m)\left(\frac{d q}{d k}(a)\right)=\frac{d}{d k}{ }_{\mid k=a} \mathcal{H}\left(p \gamma_{\alpha}^{m} q_{\mid[k, a]}{ }^{-1} \gamma_{\alpha}^{q(k)^{-1}} p^{-1}\right)
$$

where $q_{[[k, a]}{ }^{-1}$ is to be read as $q_{\mid[a, k]}$ whenever $a \leq k$.

The reasoning we shall be using in the next section requires us to find a similar formula for a larger family of gauge potentials. These will be obtained through the pull-back of the connection 1-form $\omega$ by further local sections of the form $s_{\alpha, g}=s_{\alpha} . g, g \in G$. The new potentials shall be referred to as $\Lambda_{\alpha, g}=s_{\alpha, g}^{*} \omega$.

Proposition 1. The vector space generated by the union of images

$$
\bigcup_{\alpha} \bigcup_{g \in G} \bigcup_{m \in U_{\alpha}} \operatorname{Im}\left(\Lambda_{\alpha, g}(m)\right)
$$

is a subalgebra of $\mathcal{G}$. When $g \in G$ is a holonomy value the potential $\Lambda_{\alpha, g}$ may be expressed by the formula

$$
\Lambda_{\alpha, \mathcal{H}(l)}(m)\left(\frac{d q}{d k}(a)\right)=\frac{d}{d k}{ }_{k=a} \mathcal{H}\left(l^{-1} p \gamma_{\alpha}^{m} q_{\mid[k, a]}{ }^{-1} \gamma_{\alpha}^{q(k)^{-1}} p^{-1} l\right)
$$

where all the paths are to be understood as previously explained in this section.

Proof: The gauge transformation law tells us that $\Lambda_{\alpha, h}=$ $\operatorname{Ad}\left(h^{-1} g\right) \cdot \Lambda_{\alpha, g}+\left(g^{-1} h\right)^{*} \theta$ where $\theta$ is the canonical 1-form of $G$ and $g^{-1} h$ is regarded as a constant function defined on $U_{\alpha}$. Obviously this equation reduces to $\Lambda_{\alpha, h}=\mathbf{A d}\left(h^{-1} g\right) . \Lambda_{\alpha, g}$ which shows that the vector space mentioned in the proposition is invariant under the adjoint representation of $G$ and, therefore, is a subalgebra of $\mathcal{G}$. The transformation $\Lambda_{\alpha, \mathcal{H}(l)}=\mathbf{A d}\left(\mathcal{H}(l)^{-1}\right) \cdot \Lambda_{\alpha}$ shows how to calculate $\Lambda_{\alpha, \mathcal{H}(l)}$ given a formula for $\Lambda_{\alpha}$ :

$$
\begin{aligned}
\Lambda_{\alpha, \mathcal{H}(l)}(m)\left(\frac{d q}{d k}(a)\right) & =\operatorname{Ad}\left(\mathcal{H}(l)^{-1}\right) \cdot \frac{d}{d k}{ }_{\mid k=a} \mathcal{H}\left(p \gamma_{\alpha}^{m} q_{\mid[k, a]}{ }^{-1} \gamma_{\alpha}^{q(k)^{-1}} p^{-1}\right) \\
& =\frac{d}{d k}{ }_{\mid k=a} \mathcal{H}(l)^{-1} \mathcal{H}\left(p \gamma_{\alpha}^{m} q_{\mid[k, a]}{ }^{-1} \gamma_{\alpha}^{q(k)^{-1}} p^{-1}\right) \mathcal{H}(l) \\
& =\frac{d}{d k}{ }_{\mid k=a} \mathcal{H}\left(l^{-1} p \gamma_{\alpha}^{m} q_{\mid[k, a]}{ }^{-1} \gamma_{\alpha}^{q(k)^{-1}} p^{-1} l\right) .
\end{aligned}
$$




\section{The kernel theorem}

Proposition 2. If the connection $\nabla$ reconstructed from the holonomy $\mathcal{H}: \mathcal{G} \mathcal{L}^{\infty}(M) \rightarrow G$ is irreducible then the gauge potentials of Proposition 1 are such that the aforementioned vector space generated by the union of images

$$
\bigcup_{\alpha} \bigcup_{g \in G} \bigcup_{m \in U_{\alpha}} \operatorname{Im}\left(\Lambda_{\alpha, g}(m)\right)
$$

is the whole Lie algebra $\mathcal{G}$ associated with $G$.

Proof: If $\nabla$ is an irreducible $G$-connection then the holonomy $\mathcal{H}_{\nabla}$ is surjective, otherwise $\nabla$ could be further reduced. In this context the Ambrose-Singer theorem states that the set of all curvature values $\left\{\Omega_{b}(x, y): b \in B \wedge x, y \in T_{b} B\right\}$ generates the Lie algebra $\mathcal{G}$. This generator set is equal to $\left\{\left(s_{\alpha, g}^{*} \Omega\right)_{m}(x, y): g \in G, m \in U_{\alpha} \wedge x, y \in T_{m} M\right.$, for some $\alpha$ \} because the image of each subfamily of differentials $D\left(s_{\alpha, g}\right)$, $g \in G$, forms a distribution on $\pi^{-1}\left(U_{\alpha}\right)$ which is in direct sum with the vertical distribution of the bundle. The field strengths $\Omega_{\alpha, g}=s_{\alpha, g}^{*} \Omega$ are related to the gauge potentials $\Lambda_{\alpha, g}=s_{\alpha, g}^{*} \omega$ by

$$
\Omega_{\alpha, g}=\mathbf{d} \Lambda_{\alpha, g}+\frac{1}{2}\left[\Lambda_{\alpha, g}, \Lambda_{\alpha, g}\right]
$$

so that the union of images of all 2-forms $\mathbf{d} \Lambda_{\alpha, g}+\frac{1}{2}\left[\Lambda_{\alpha, g}, \Lambda_{\alpha, g}\right]$ is known to generate $\mathcal{G}$. The definition of the exterior derivative $\mathbf{d}$ makes sure that all values generated by vectors $\left(\mathbf{d} \Lambda_{\alpha, g}\right)_{m}(x, y)$ must be generated by vectors $\Lambda_{\alpha, g}(m)(x)$ and a similar remark can be made about the remaining term $\frac{1}{2}\left[\Lambda_{\alpha, g}, \Lambda_{\alpha, g}\right]$ because of Proposition 1 .

Proposition 3. If $\mathcal{H}_{1}: \mathcal{G L}^{\infty}(M) \rightarrow G_{1}$ and $\mathcal{H}_{2}: \mathcal{G} \mathcal{L}^{\infty}(M) \rightarrow G_{2}$ are surjective holonomies which share the same kernel, then $G_{1}$ and $G_{2}$ are isomorphic as Lie groups.

Proof: $\hat{\mathcal{H}}_{1}$ and $\hat{\mathcal{H}}_{2}$ are group isomorphisms obtained from $\mathcal{H}_{1}$ and $\mathcal{H}_{2}$ quotienting by their respective kernels, which are identical. $\mathcal{F}=$ $\hat{\mathcal{H}}_{2} \circ \hat{\mathcal{H}}_{1}^{-1}$ is a group isomorphism. Now, Propositions 1 and 2 show that there exist indices $\alpha_{1}, \alpha_{2}, \ldots, \alpha_{k}$, loops $l_{1}, l_{2}, \ldots, l_{k}$, paths $p_{1}, p_{2}, \ldots, p_{k}, q_{1}, q_{2}, \ldots, q_{k}$ and instants $a^{1}, a^{2}, \ldots, a^{k}$, such that for

$$
\psi_{i}(s)=l_{i}^{-1} p_{i} \gamma_{\alpha_{i}}^{q_{i}\left(a^{i}\right)} q_{i \mid\left[s, a^{i}\right]}{ }^{-1} \gamma_{\alpha_{i}}^{q_{i}(s)^{-1}} p_{i}^{-1} l_{i}
$$

defined on a neighborhood of $a^{i}$ and where $q_{i \mid\left[s, a^{i}\right]}{ }^{-1}$ is to be read as $q_{i \mid\left[a^{i}, s\right]}$ whenever $a^{i} \leq s$, the vectors

$$
v_{i}=\frac{d}{d s}{ }_{\mid s=a^{i}} \mathcal{H}_{1}\left(\psi_{i}(s)\right)
$$


form a basis $v_{1}, v_{2}, \ldots, v_{k}$ for the Lie algebra associated with $G_{1}$. Consider the following function defined on a neighborhood $A \subseteq] 0,1\left[^{k}\right.$ of $\left(a^{1}, a^{2}, \ldots, a^{k}\right)$ by a product of loops:

$$
\begin{aligned}
& \Psi: A \subseteq] 0,1\left[{ }^{k} \rightarrow \mathcal{G} \mathcal{L}^{\infty}(M)\right. \\
& \Psi\left(s^{1}, s^{2}, \ldots, s^{k}\right)=\psi_{1}\left(s^{1}\right) \psi_{2}\left(s^{2}\right) \ldots \psi_{k}\left(s^{k}\right) .
\end{aligned}
$$

About $\left.\mathcal{H}_{1} \circ \Psi: A \subseteq\right] 0,1\left[{ }^{k} \rightarrow G\right.$ we know that:

a) $\mathcal{H}_{1} \circ \Psi$ is smooth

b) $\mathcal{H}_{1} \circ \Psi\left(a^{1}, a^{2}, \ldots, a^{k}\right)=\mathbf{e} \in G$

c) $\mathcal{H}_{1} \circ \Psi\left(s^{1}, s^{2}, \ldots, s^{k}\right)=\mathcal{H}_{1} \circ \psi_{1}\left(s^{1}\right) \mathcal{H}_{1} \circ \psi_{2}\left(s^{2}\right) \ldots \mathcal{H}_{1} \circ \psi_{k}\left(s^{k}\right)$

d) $\frac{\partial\left(\mathcal{H}_{1} \circ \Psi\right)}{\partial s^{i}}\left(a^{1}, a^{2}, \ldots, a^{k}\right)=v_{i}$.

The inverse function theorem guarantees that there exist open neighborhoods $A^{\prime} \subseteq A$ of $\left(a^{1}, a^{2}, \ldots, a^{k}\right)$ and $B \subseteq G_{1}$ of $\mathbf{e} \in G_{1}$, such that the restriction $\left.\mathcal{H}_{1} \circ \Psi_{\mid A^{\prime}}: A^{\prime} \subseteq\right] 0,1\left[{ }^{k} \longrightarrow B \subseteq G_{1}\right.$ is a diffeomorphism. Bringing back the algebraic isomorphism $\mathcal{F}=\hat{\mathcal{H}}_{2} \circ \hat{\mathcal{H}}_{1}^{-1}$ it is easy to check that $\mathcal{F}_{\mid B}=\left(\mathcal{H}_{2} \circ \Psi\right) \circ\left(\mathcal{H}_{1} \circ \Psi\right)_{\mid A^{\prime}}^{-1}$ and that $\mathcal{F}: G_{1} \rightarrow G_{2}$ is smooth. Exchanging $G_{1}$ and $G_{2}$ one shows that the inverse $\mathcal{F}^{-1}$ is also smooth and, therefore, $\mathcal{F}$ is a Lie group isomorphism.

Theorem 2. If two irreducible connections $\nabla_{1}$ and $\nabla_{2}$, with structural groups $G_{1}$ and $G_{2}$, define holonomies $\mathcal{H}_{1}: \mathcal{G L}^{\infty}(M) \rightarrow G_{1}$ and $\mathcal{H}_{2}: \mathcal{G L}^{\infty}(M) \rightarrow G_{2}$, which share the same kernel, then there exists a principal bundle isomorphism that carries $\nabla_{1}$ into $\nabla_{2}$.

Proof: The two connections being irreducible means that their holonomies are surjective. So Proposition 3 applies and there is a group isomorphism $\mathcal{F}: G_{1} \rightarrow G_{2}$. This allows us to define a map $\hat{\mathcal{F}}$ on the bundle reconstructed from $\mathcal{H}_{1}$ to the bundle reconstructed from $\mathcal{H}_{2}$ by means of $\hat{\mathcal{F}}\left([(p, g)]_{1}\right)=[(p, \mathcal{F}(g))]_{2}$. To check that $\hat{\mathcal{F}}$ is well defined one must recall that $\mathcal{F}=\hat{\mathcal{H}}_{2} \circ \hat{\mathcal{H}}_{1}^{-1}$ and take into account the equivalence relation used to reconstruct bundles. $\hat{\mathcal{F}}$ establishes a bijection between both reconstructed bundles that is compatible with the $G$-right actions and carries horizontal lifts, with regard to $\nabla_{1}$, into horizontal lifts with respect to $\nabla_{2}$. The smoothness of $\hat{\mathcal{F}}$ and its inverse is ensured by the smoothness of $\mathcal{F}$ and $\mathcal{F}^{-1}$.

\section{Generalized holonomies}

In some recent approaches to quantization of Yang-Mills and gravity theories, certain authors $([\mathbf{A I}],[\mathbf{A L}],[\mathbf{L}],[\mathbf{M M}])$ have been led to consider objects which generalize the notions of connection and holonomy by no longer insisting on smoothness. In particular one defines 
Definition 2. A generalized holonomy is a group morphism $\mathcal{H}$ : $\mathcal{G} \mathcal{L}^{\infty}(M) \rightarrow G$.

Whilst the image of a holonomy, known as the holonomy group, is always a Lie subgroup of $G$ (not necessarily closed), this may no longer hold for generalized connections. By applying a theorem of Kuranishi and Yamabe on arcwise-connected subgroups we can at least make the following statement:

Theorem 3. If a generalized holonomy $\mathcal{H}: \mathcal{G} \mathcal{L}^{\infty}(M) \rightarrow G$ is continuous in the sense that the composition

$$
[0,1] \subset \mathbf{R}^{1} \stackrel{\psi}{\longrightarrow} \Omega^{\infty}(M) \stackrel{\text { proj }}{\longrightarrow} \mathcal{G L}^{\infty}(M) \stackrel{\mathcal{H}}{\longrightarrow} G
$$

where proj is the natural projection, is continuous throughout the interval $[0,1] \subseteq \mathbf{R}^{1}$ for every smooth family of loops $\psi:[0,1] \subseteq \mathbf{R}^{1} \rightarrow \Omega^{\infty}(M)$, then the image $\operatorname{Im} \mathcal{H}$ is a Lie subgroup of $G$ (not necessarily closed).

Proof: Because intimacy is stronger then usual homotopy there arises a canonical epimorphism $\mathcal{C}: \mathcal{G} \mathcal{L}^{\infty}(M) \rightarrow \pi_{1}(M)$. Let us consider the normal subgroup $\operatorname{ker}(\mathcal{C}) \subseteq \mathcal{G L}^{\infty}(M)$. The image $\mathcal{H}(\operatorname{ker}(\mathcal{C}))$ is a normal subgroup of $\operatorname{Im} \mathcal{H}$ and $\mathcal{H}$ goes to the quotient to define an epimorphism from $\mathcal{G L}^{\infty}(M) / \operatorname{ker}(\mathcal{C})$ onto $\operatorname{Im} \mathcal{H} / \mathcal{H}(\operatorname{ker}(\mathcal{C}))$. It follows that $\operatorname{Im} \mathcal{H} / \mathcal{H}(\operatorname{ker}(\mathcal{C}))$ is countable because $\mathcal{C}$ goes to the quotient to define an isomorphism between $\mathcal{G} \mathcal{L}^{\infty}(M) / \operatorname{ker}(\mathcal{C})$ and $\pi_{1}(M)$ and this last set is countable due to the topology of $M$ being second countable. So it will be enough to show that $\mathcal{H}(\operatorname{ker}(\mathcal{C}))$ is a Lie subgroup of $G$. Due to a theorem of Kuranishi and Yamabe (see $[\mathbf{K N}]$ and $[\mathbf{Y}]$ ) we just have to show that $\mathcal{H}(\operatorname{ker}(\mathcal{C}))$ is arcwise connected. Given $g \in \mathcal{H}(\operatorname{ker}(\mathcal{C}))$ there is a loop $l \in \Omega^{\infty}(M)$ whose generalized holonomy is $\mathcal{H}(l)=g$ and there is a smooth homotopy $\psi:[0,1] \subseteq \mathbf{R}^{1} \rightarrow \Omega^{\infty}(M)$ such that $\psi(0)=l$ and $\psi(1)$ is the trivial loop. Obviously $\mathcal{H} \circ \psi$ is a continuous curve contained in $\mathcal{H}(\operatorname{ker}(\mathcal{C}))$ and joining $g$ and the identity.

Acknowledgements. We are indebted to Jerzy Lewandowski and John Barrett for exchange of correspondence. We also thank Roger Picken, José Mourão, João Nuno Tavares, Robin W. Tucker and Armando Machado for many useful conversations.

\section{References}

[AI] A. Ashtekar and C. J. Isham, Representations of the holonomy algebras of gravity and non-Abelian gauge theories, Classical Quantum Gravity 9 (1992), 1433-1467. 
[AL] A. Ashtekar And J. Lewandowski, Representation theory of analytic holonomy $C^{*}$-algebras, in "Knots and Quantum Gravity," J. Baez Ed., Oxford University Press, 1994.

[Ba] J. W. BARRETT, Holonomy and path structures in general relativity and Yang-Mills Theory, Internat. J. Theoret. Phys. 30 (1991), 1171-1215.

[CP] A. Caetano And R. Picken, Internat. J. Math. 5 (1994), 835-848.

[KN] S. Kobayashi AND K. Nomizu, "Foundations of Differential Geometry," Interscience Publishers, 1963.

[L] J. LEWANDOWski, The group of loops, holonomy maps, path bundle and path connection, Classical Quantum Gravity 10 (1993), 879-904.

[MM] D. Marolf And J. M. MourÃo, On the carrier of the Ashtekar-Lewandowski measure, Comm. Math. Phys., to appear.

[Y] H. YAmABE, On an arcwise connected subgroup of a Lie group, Osaka J. Math. 2 (1950), 13-14.

Keywords. holonomy, homotopy, loop spaces

1991 Mathematics subject classifications: 53C05, 55P10, 55P35

\author{
Departamento de Matemática \\ Faculdade de Ciências \\ Universidade de Lisboa \\ R. Ernesto de Vasconcelos, Bloco C1 \\ 1700 Lisboa \\ PORTUGAL
}

Rebut el 17 de Novembre de 1995 